\title{
Surface modification of nano-TiN by using silane coupling agent
}

\author{
GuOJun ChenG $^{1,2}$, JiAqi LuO $^{1}$, JiASHENG QIAN $^{1 *}$, Jibin MiaO $^{1}$ \\ ${ }^{1}$ School of Chemistry and Chemical Engineering, Anhui University, Hefei 230039, China \\ ${ }^{2}$ School of Materials Science and Engineering, Anhui Uniersity of Science and Technology, Huainan 232001, China
}

\begin{abstract}
Titanium nitride (TiN) nano-particles were subjected to graft modification by silane coupling agent (KH-570) via a direct blending method. The hydroxyl groups on the surface of TiN nano-particles can interact with silanol groups $\left[-\mathrm{Si}-\mathrm{OCH} \mathrm{H}_{3}\right]$ of KH-570 forming an organic coating layer. The covalent bonds (Ti-O-Si) formation was testified by Fourier transform infrared spectra (FTIR) and X-ray photoelectron spectroscopy (XPS). Through transmission electron micrograph (TEM) observations, it was found that $\mathrm{KH}-570$ could improve the dispersibility of nano-TiN particles in ethyl acetate. Thermo gravimetric analysis (TGA) and contact angle measurements indicated that KH-570 molecules were adsorbed or anchored on the surface of nano-TiN particle and the net efficiency of it was $22.76 \%$, which facilitated to hinder the aggregation of nano-TiN particles.
\end{abstract}

Keywords: nano titanium nitride; silane coupling agents; surface modification; dispersion

(C) Wroclaw University of Technology.

\section{Introduction}

In recent years, nano-particles have received considerable attention for their special physical and chemical properties. However, inorganic nano-particles are very prone to agglomerate in media and show poor dispersion capacity in organic solvents and oil due to their high surface energy. Because of the inconsistent interfacial interaction, the combination of the inorganic nano-particles with the polymer matrix is weak, which limits the wide application of nano-particles [1-3]. How to disperse the inorganic nano-particles in organic polymer matrices and improve their interfacial interaction are the main issues to be solved. Fortunately, this problem can be resolved by using some special preparation techniques, for example, the preparation of nano-particles with surface modified by organic compounds. If the surface modification agents are high-weight hydrocarbons, the nano-particles can be dispersed stably in organic solvents and oil [46]. It has been reported that organic compound

*E-mail: qianjs@ahu.edu.cn coatings on $\mathrm{TiO}_{2}, \mathrm{CeO}_{2}, \mathrm{SiO}_{2}$ and $\mathrm{Al}_{2} \mathrm{O}_{3}$ have been synthesized successfully to improve their dispersive capacity in organic solvents and polymer matrices [7-12]. However, few of these papers have studied systemically the surface modification of nano-titanium nitride (TiN) with organic silane coupling agents.

TiN particles are widely used due to their superior properties, such as good wear resistance, high melting point $\left(2950{ }^{\circ} \mathrm{C}\right)$, high hardness, high chemical and thermal stability, high corrosion resistance, high electrical and thermal conductivity $\left(5 \times 10^{4} \Omega^{-1} \mathrm{~cm}^{-1}\right)$, biocompatibility and antimicrobial effect for medical devices and surgical tools [13-19]. $\gamma$-methacryloxypropyl trimethoxy silane (KH-570), as a silane coupling agent, is often used to modify the fillers such as $\mathrm{ZnO}, \mathrm{TiO}_{2}$, carbon fiber, and so on. When the modified fillers are introduced into a certain polymer matrix, the interface adhesion can be enhanced significantly [20-23].

In the present work, some silane coupling agents were found to make nano-TiN particles disperse well and steadily in many organic mediums. In order to further study the effect 
of coupling agent on the composite interface, KH-570 was used as a modifying agent to control the surface properties of nano-TiN particles. The structural changes of the modified nano-TiN (M-TiN) particles were characterized by Fourier infrared spectra (FTIR), X-ray photoelectron spectroscopy (XPS), transmission electron microscopy (TEM). Contact angle measuring instrument (CAMI) and thermogravimetric analysis (TGA) were also used in this experiment. On the one hand, the side groups $\left[-\mathrm{Si}\left(\mathrm{OCH}_{3}\right)_{3}\right]$ in the main chain of $\mathrm{KH}-570$ may react with $-\mathrm{OH}$, $-\mathrm{NH}$ and $-\mathrm{NH}_{2}$ on the surface of the nano-TiN powder containing active hydrogen. On the other hand, KH-570 has a carbon chain structure and can provide great compatibility with organic solvents and polymer matrices. This paper attempts to characterize the performances of the M-TiN particles, and analyze the potential application of the M-TiN to materials. It can help to design high performance composite materials.

\section{Experimental}

\subsection{Surface modification of nano-TiN}

TiN nano-particles used in this experiment were commercial ultra-fine powders with the following characteristics: the average particle diameter $25.0 \mathrm{~nm}$, the specific surface area $-115 \mathrm{~m}^{2} \mathrm{~g}^{-1}$. All of the reagents were analytical-grade and used as received. Before modification, pristine TiN nano-particles were dried at $110.0{ }^{\circ} \mathrm{C}$ in a vacuum oven for $10 \mathrm{~h}$ to remove the moisture adsorbed on their surface. Then, TiN nano-particles $(1.0 \mathrm{~g})$ were dispersed in $150.0 \mathrm{ml}$ ethyl acetate solvent by sonication, and $0.33 \mathrm{~g}$ of $\mathrm{KH}-570$ was added. The mixture was refluxed at $75.0{ }^{\circ} \mathrm{C}$ for $3.5 \mathrm{~h}$. After the reaction, the nano particles were separated by centrifuging, washed with ethyl acetate for some time and then dried at $50{ }^{\circ} \mathrm{C}$ for $24 \mathrm{~h}$ in a vacuum oven in order to remove the ethyl acetate.

\subsection{Structural characterization}

To evaluate the surface chemistry, FTIR spectra of nano-TiN and M-TiN were obtained with the use of a spectrometer (Nicolet Co., Nexus-870,

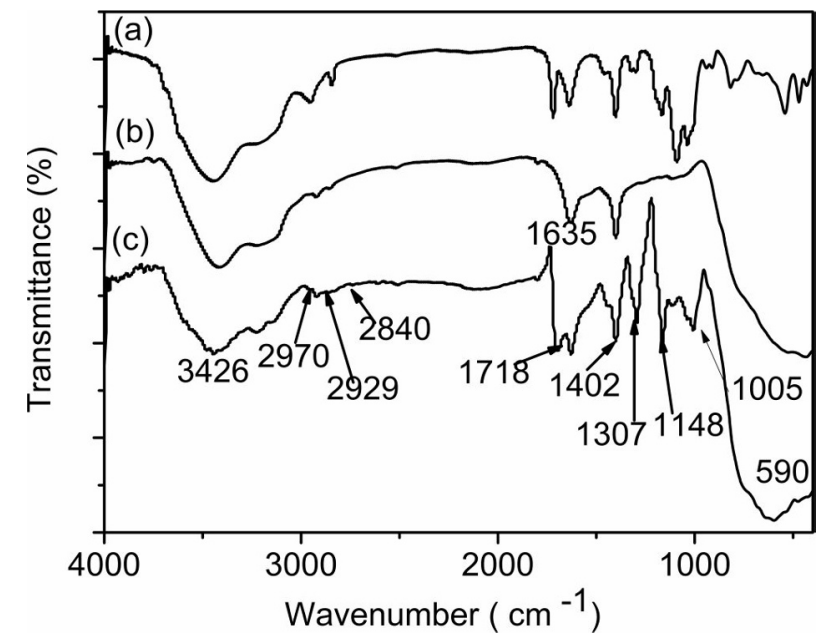

Fig. 1. FTIR spectra of (a) KH-570, (b) pristine nano-TiN, (c) M-TiN.

USA). The FTIR spectra were recorded using the potassium bromide pellets of the samples and analyzed from $400 \mathrm{~cm}^{-1}$ to $4000 \mathrm{~cm}^{-1}$ with a $4 \mathrm{~cm}^{-1}$ resolution.

Composition of nano-TiN before and after surface modification was determined by XPS (VG Scientific Ltd., ESCALAB-MK-II, England) with $\mathrm{Al} \mathrm{K} \alpha \mathrm{X}$-ray $(\mathrm{h} v=1486.6 \mathrm{eV})$ at $15.0 \mathrm{kV}$ and 150.0 W.

\subsection{Particles characterization}

TEM images were obtained to characterize the structures of samples. TEM observations of the samples morphology were performed using JEM-2100 TEM (JEOL, Japan) at $200 \mathrm{kV}$.

The contact angle was investigated using CAMI (Kino Co., SL 200C, USA) at the temperature of $25.0{ }^{\circ} \mathrm{C}$. The surfaces of the samples were dropped by water and the angle value was taken as its contact angle.

The content of the intercalated modifier was determined by TGA with a thermal analyzer (Netzsch Co., STA449F3, Germany). Samples were heated to $600{ }^{\circ} \mathrm{C}$ from room temperature at a rate of $20^{\circ} \mathrm{C} \mathrm{min}-1$. 


\section{Results and discussion}

\subsection{Structural characterization}

FTIR technique was used to show the structural changes in TiN nano-particles modified by coupling agent. Fig. 1 shows the FTIR spectra of pristine TiN and M-TiN modified with KH-570. The characteristic adsorption peaks of carboxyl $(\mathrm{C}=\mathrm{O})$ at $1720 \mathrm{~cm}^{-1},-\mathrm{Si}-\mathrm{OH}$ at $1300 \mathrm{~cm}^{-1}$, $-\mathrm{Si}-\mathrm{O}-\mathrm{C}$ at $1080 \mathrm{~cm}^{-1}$ and $-\mathrm{CH}_{3}$ in the region of $2800-3000 \mathrm{~cm}^{-1}$ belong to KH-570 from Fig. 1a [12]. Fig. 1b and Fig. 1c show typical FTIR spectra of pristine and M-TiN particles modified with KH-570, respectively. Nano-TiN is highly reactive and easy to be oxidized upon exposure to air. So, the surface region of commercial nano-TiN particles has a large amount of $\mathrm{Ti}-\mathrm{NH}_{2}$, and Ti-OH groups. Fig. 1b shows the FTIR spectrum of pristine $\mathrm{TiN} ; v_{\mathrm{O}-\mathrm{H}}$ and $v_{\mathrm{N}-\mathrm{H}}$ combination bands can be seen at $3426 \mathrm{~cm}^{-1}$ [2]. The peak at $1635 \mathrm{~cm}^{-1}$ is assigned to the $-\mathrm{NH}$ shear vibration and the asymmetric stretching vibration of it is $1000 \mathrm{~cm}^{-1}$, whereas the absorption peak near $600 \mathrm{~cm}^{-1}$ corresponds to the Ti-N absorption bands [24]. From the FTIR spectra of M-TiN, we can see that it is similar to that of KH-570. The absorption peaks at $2800 \mathrm{~cm}^{-1}, 3000 \mathrm{~cm}^{-1}$, $1718 \mathrm{~cm}^{-1}, 1635 \mathrm{~cm}^{-1}$, and $1000-1200 \mathrm{~cm}^{-1}$ are attributed to the $-\mathrm{CH}_{2}-,-\mathrm{CH}_{3}, \mathrm{C}=\mathrm{O}, \mathrm{C}=\mathrm{C}$ and $\mathrm{Si}-\mathrm{O}$ groups, respectively. It can be found that the peak of $\mathrm{Si}-\mathrm{O}$ is broader and stronger than that of the KH-570 in $1000-1150 \mathrm{~cm}^{-1}$. This indicates that the surface group of nano-TiN has changed from Ti-OH to Ti-O-Si-C. The coupling agent is tightly adsorbed on the surface of the nano-TiN by chemisorption [2, 25, 26]. Although the FTIR spectra have unambiguously indicated the successful functionalization of KH-570 to the nano-TiN surface, the findings were additionally confirmed by XPS. Fig. 2 displays XPS spectra of nano-TiN after surface modification where the binding energy of $\mathrm{C} 1 \mathrm{~s}(284.9 \mathrm{eV})$ is used as the reference. The high resolution $\mathrm{N}$ 1s spectrum (Fig. 2A) of pristine nano-TiN occurs at $396.4 \mathrm{eV}$, which can be attributed to Ti-N component. The Ti spectrum displays four peaks at $563.1 \mathrm{eV}, 458.4 \mathrm{eV}$, $60.5 \mathrm{eV}$ and $36.2 \mathrm{eV}$, which can be ascribed to
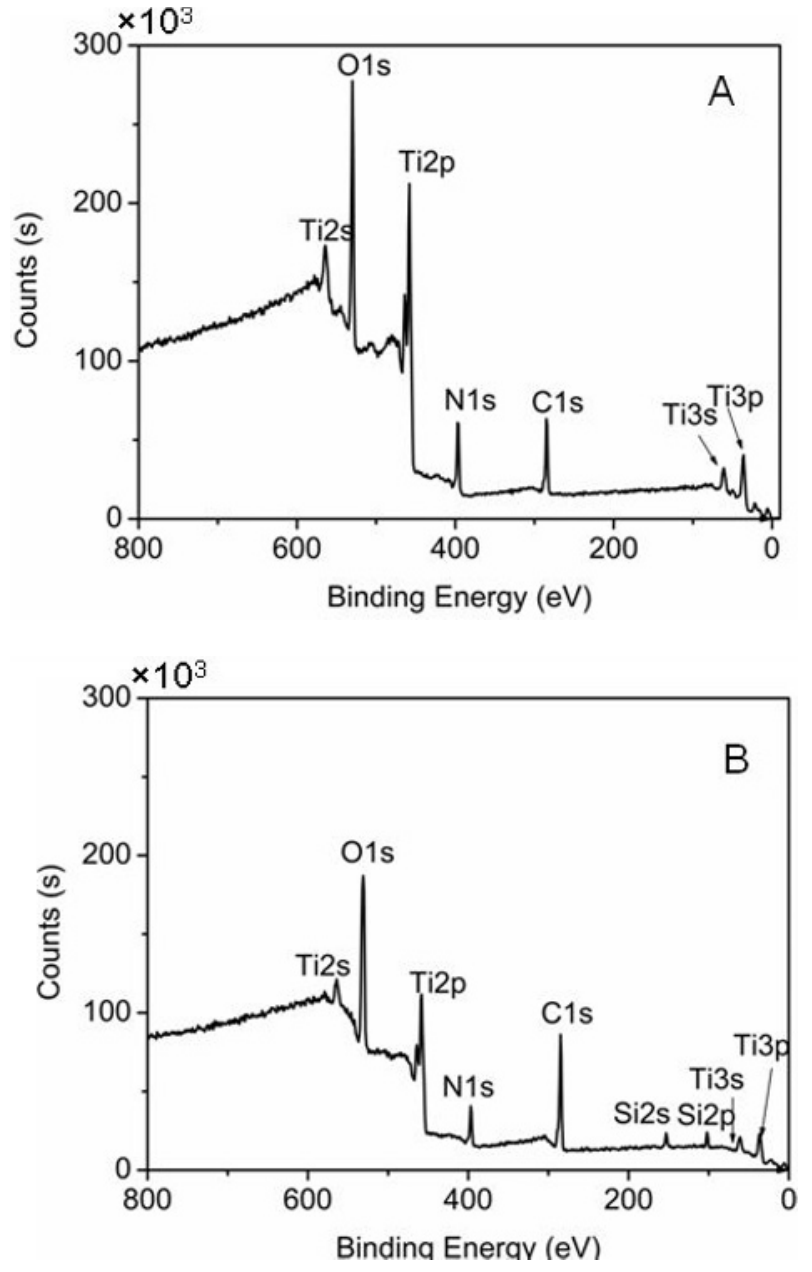

Fig. 2. XPS spectra for (A) native TiN and (B) M-TiN particles.

$\mathrm{Ti}-\mathrm{O}, \mathrm{Ti}-\mathrm{N}$ and $\mathrm{Ti}-\mathrm{N}-\mathrm{O}$, respectively $[15,27]$. The $\mathrm{O}$ and $\mathrm{C}$ spectra display peaks at $530.1 \mathrm{eV}$ and $284.5 \mathrm{eV}$, which correspond to Ti-OH and surface contamination by carbon component of nano-TiN, respectively [28, 29]. In the XPS spectrum of $\mathrm{M}-\mathrm{TiN}$ (Fig. 2B), in addition to nitrogen ( $\mathrm{N}$ 1s $396.8 \mathrm{eV}$ ), titanium (Ti 2s $564.7 \mathrm{eV}$, Ti 2p $459.2 \mathrm{eV}$, Ti 3s $61.1 \mathrm{eV}$ and Ti 3p $36.4 \mathrm{eV}$ ), carbon (C 1s $284.9 \mathrm{eV}$ ) and oxygen (O 1s $531.3 \mathrm{eV}$ ) photoelectron peaks, there are also silicon ( $\mathrm{Si}$ 2s $153.0 \mathrm{eV}$ and Si 2p $101.9 \mathrm{eV}$ ) photoelectron peaks [15]. The appearance of the peaks at $531.3 \mathrm{eV}, 153.0 \mathrm{eV}$ and $101.9 \mathrm{eV}$ (corresponding to oxygen and silicon in $\mathrm{Ti}-\mathrm{O}-\mathrm{Si}$ ) after surface modification indicates the chemical bonding between KH-570 and titanium oxide [30, 31]. 

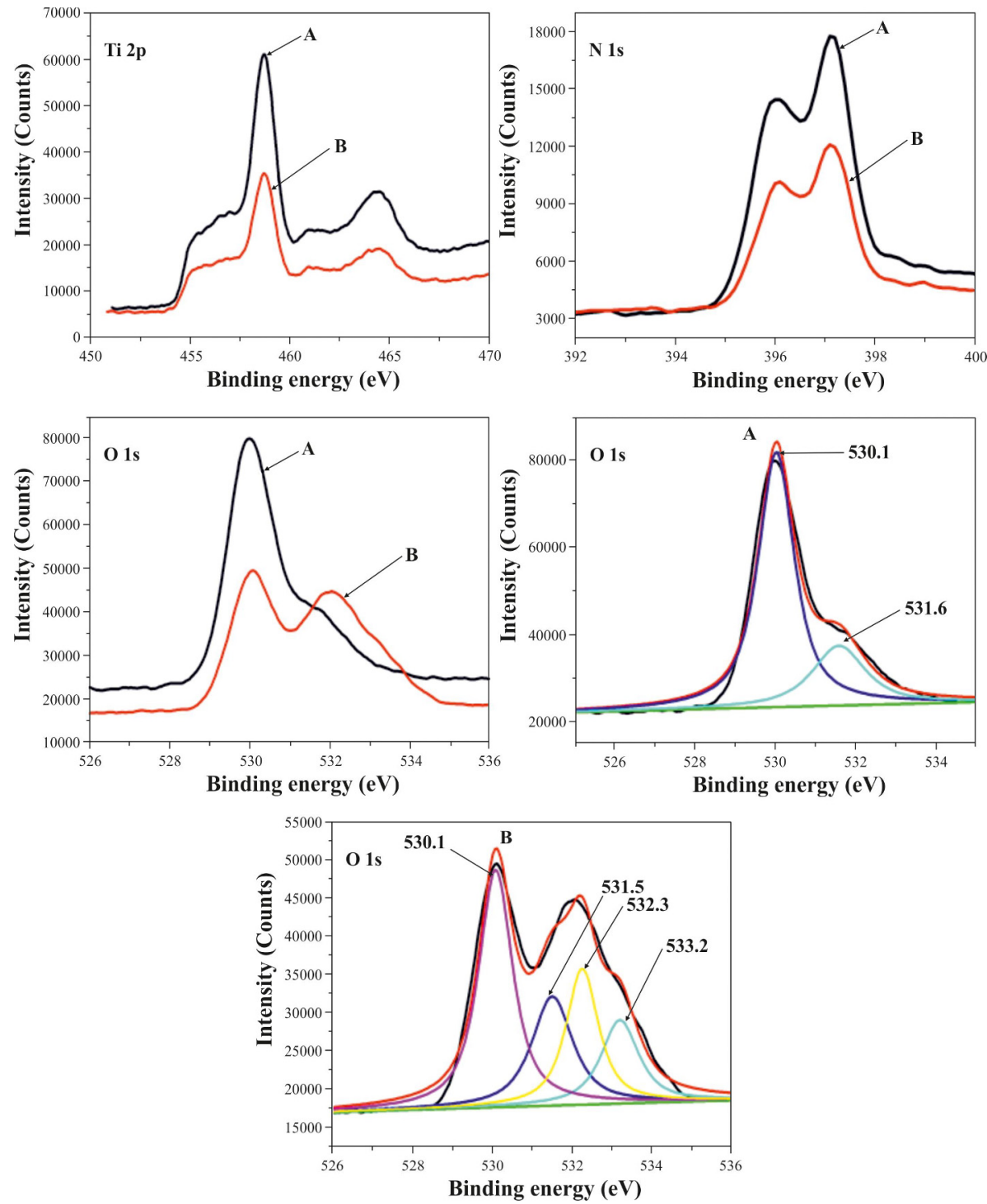

Fig. 3. Ti 2p, N 1s and $\mathrm{O} 1 \mathrm{~s}$ XPS fine scan spectra and the multiple sub-peaks of $\mathrm{O}$ 1s and $\mathrm{Si} 2 \mathrm{p}$ using Gaussian-Lorentzian fit for nano-TiN (A: pristine, B: modified)

Fig. 3 shows the Ti 2p, $\mathrm{N}$ 1s and $\mathrm{O}$ 1s peaks and the simultaneous splitting of $\mathrm{C} 1 \mathrm{~s}$ core levels spectra. It can be found that the M-TiN's Ti $2 p, N$ $1 \mathrm{~s}$ and $\mathrm{O} 1 \mathrm{~s}$ peak areas (content of carbon element), peak shapes and positions have changed, which reflects the chemical interaction between nano-TiN and graft KH-570. From Fig. 3, it can be seen that the typical $\mathrm{O} 1 \mathrm{~s}$ spectra (at $531.8 \mathrm{eV}$ and $530.1 \mathrm{eV}$ ) indicate that there are abundant oxygen of $\mathrm{TiN}$ or Ti-OH bonds in the unmodified nano-TiN samples. After being modified, the binding energy of the $\mathrm{O}$ 1s peak changes from 531.8 to $532.3 \mathrm{eV}$ and
$533.2 \mathrm{eV}$ for Ti-O-Si. All data of XPS suggest that KH-570 covalently bonds to the surface of nanosized TiN particles and an organic coating layer is formed. This result is in agreement with that obtained from FTIR.

\subsection{Particles characterization}

Surface hydrophilicity of nano-TiN particles was investigated by contact angle measurements as shown in Fig. 4. After surface modification, the contact angle has increased from $21.15^{\circ}$ 


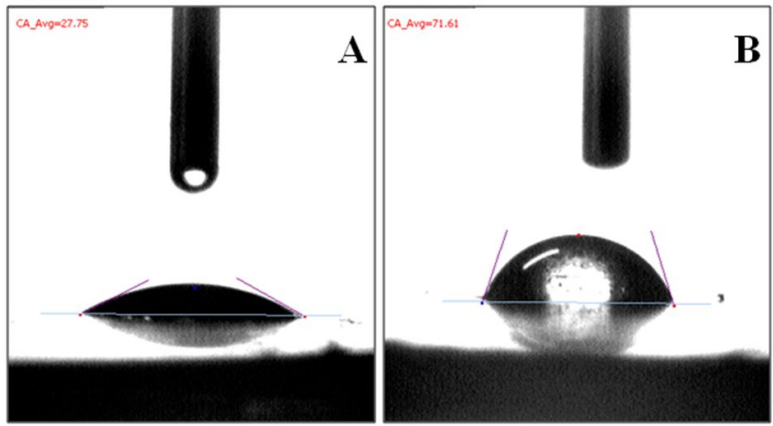

Fig. 4. Contact angle images of (A) nano-TiN and (B) M-TiN particles.

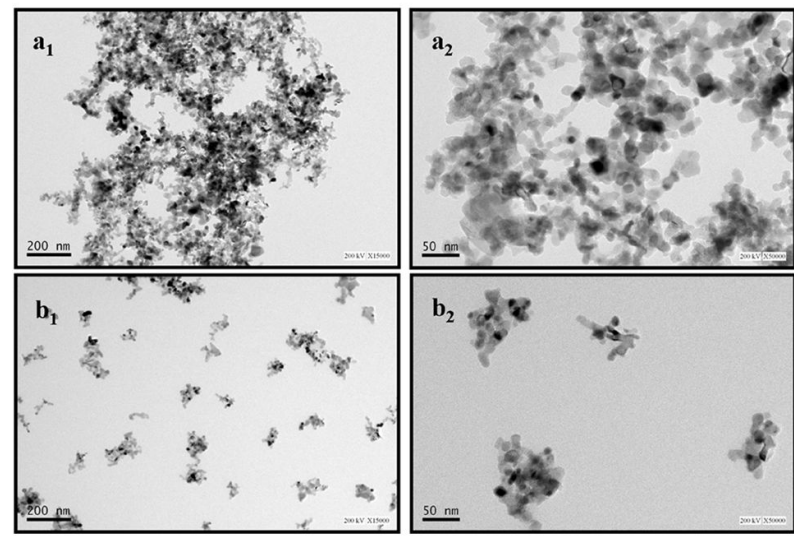

Fig. 5. TEM images for (a) nano-TiN and (b) M-TiN particles.

to $71.61^{\circ}$ in water, which suggests that the surface hydrophobicity of nano-TiN nano-particles increased while the surface free energy of it decreased. These changes are likely to be due to the hydrophobic carbon backbone of the graft coupling agent [2, 32]. It can be inferred that after modification with $\mathrm{KH}-570$, the nano-TiN particles can be dispersed in polymer materials easier than pristine nano-TiN particles. Thus, the M-TiN can be dispersed more easily in non-polar or weak polar polymer materials than pristine nano-TiN particles. Fig. 5 displays the TEM images of native nano-TiN and modified nano-TiN suspensions in ethyl acetate. In order to present detailed morphological information of the samples,

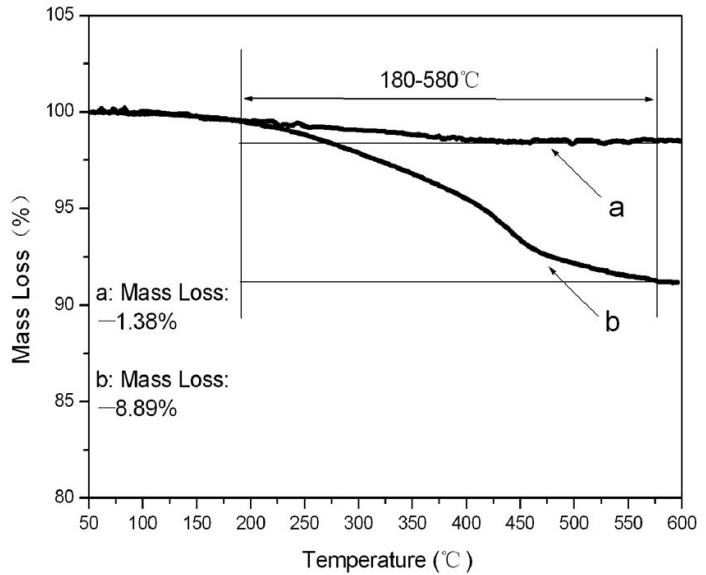

Fig. 6. TGA curves of (a) pristine nano-TiN and (b) M-TiN particles.

different magnifications are utilized for various samples. The obvious agglomerations can be seen in the images of native nano-TiN (Fig. 5a) and the homogeneous dispersion can be observed in the images of modified nano-TiN (Fig. 5b). This suggests that physical or chemical bonding occurs between the polar bonds of KH-570 and hydroxide groups of nano-TiN. The coupling agent chains grafted on the surface of nano-TiN cause mutual exclusion and steric hindrance effect. As a result, the surface free energy has been reduced correspondingly and the agglomeration is controlled. All the results further illustrate that KH-570 has played an important role in the dispersion of the nano-TiN particles.

\subsection{Thermostability of $n-T i N$ particles}

Thermal stability of nano-TiN was measured by using TGA and the results are shown in Fig. 6. As shown in Fig. 6a, the thermal decomposition of pristine nano-TiN begins at about $180^{\circ} \mathrm{C}$ and the continuous mass loss can be observed from $180^{\circ} \mathrm{C}$ to $580{ }^{\circ} \mathrm{C}$. This is likely to be due to a series of chemical reactions happening on the surface of the nano-TiN between surface groups or some physically and chemically adsorbed substances, followed by the desorption of $\mathrm{H}_{2} \mathrm{O}$, etc. $[1,12]$. So, the mass loss of pristine nano-TiN is $1.38 \%$. The thermal degradation curve of nano-TiN modified with $\mathrm{KH}-570$ is shown in Fig. 6b. The total weight 
loss of $8.89 \%$, from $180{ }^{\circ} \mathrm{C}$ to $580{ }^{\circ} \mathrm{C}$ can be attributed to the degradation of $\mathrm{KH}-570$ and other adsorption substances on the surface. So we can get the net efficiency of the KH-570, which is calculated as follows:

$$
\begin{aligned}
& \text { net efficiency of the } \mathrm{KH}-570= \\
& =\frac{8.89 \%-1.38 \%}{33 \%} \times 100 \%=22.76 \%
\end{aligned}
$$

Therefore, it further illustrates that the KH-570 molecular chains are anchored or grafted on the surface of nano-TiN.

\section{Conclusions}

TiN nano-particles were modified by means of surface modification in situ where silane coupling agent (KH-570) was used as a modifier. The results show that the modifier has been adsorbed on the surface of nano-TiN particles by covalent bonds and changed the surface properties of the nano-TiN material. The M-TiN particles present superior dispersivity and stability in ethyl acetate than that of the untreated ones, and the net efficiency of $\mathrm{KH}-570$ is $22.76 \%$.

\section{Acknowledgements}

This research was supported by the Research Fund for the Doctoral Program of Higher Education of China (20113401110003), the National Natural Science Foundation of China (51273001) and "211 Project" of Anhui University.

\section{References}

[1] TaI Y.L., Qian J.S., Zhang Y.C., Miao J.B., Chem. Eng. J., 141 (2008), 354.

[2] TAi Y.L., Qian J.S., MiaO J.B., Zhang Y.C., Mater. Design., 34 (2012), 522.

[3] Schmidt D.F., Giannelis E.P., Chem. Mater, 22 (2010), 167.

[4] Li Z.W., ZhU Y.F., Appl. Surf. Sci., 211 (2003), 315.

[5] Li H.Y., YAN Y., LIU B., Powder Technol., 178 (2007), 203.

[6] Sun Y.Y., Zhang Z.Q., Wong C.P., J. Colloid. Interf. Sci., 292 (2005), 436.

[7] Arita T., Moriya K.I., Yoshimura T., Minami K., NAKA T., Ind. Eng. Chem. Res., 49 (2010), 9815.

[8] Arita T., Yu U., Minami K., NaKa T., Ind. Eng. Chem. Res., 49 (2010), 1947.

[9] Kuma K.K., Raole P.M., Rayjada P.A., Chauhan N.L., Mukherjee S., Surf. Coat. Tech., 205 (2011), 187.
[10] Chen W.W., Wu S.W., Lei Y.D., Liao Z.F., Guo B.C., Liang X., Polymer, 52 (2011), 4387.

[11] Gupta S., Ramamurthy P.C., Madras G., Ind. Eng. Chem. Res., 50 (2011), 6585.

[12] Parlinska-Wojtan M., Meier S., Patscheider J., Thin Solid Films, 518 (2010), 4890.

[13] Janes R.A., Aldissi M., Kaner R.B., Chem. Mater., 15 (2003), 4431.

[14] Subramanian B., Muraleedharan C.V., AnAnthakumar R., JAYACHANDRAN M., Surf. Coat. Tech., 205 (2011), 5014.

[15] Rodríguez-ReYes J.C.F., Ni C.C., Bui H.P., Beebe T.P., Teplyakov A.V., Chem. Mater, 21 (2009), 5163.

[16] Sun D.F., Lang J.W., Yan X.B., Hu L.T., Xue Q.J., J. Solid. State. Chem., 184 (2011), 1333.

[17] Ros R.A., Dragos V.S., Appl. Surf. Sci., 258 (2012), 3871.

[18] LiU R., LUN N., QI Y.X., BAI Y.J., ZHU H.L., J. Alloy. Compd., 509 (2011), 10032.

[19] Ma S.R., Shi L.Y., FEnG X., Yu W.J., Lu B., J. Shanghai Jiaotong Univ., 3 (2008), 278.

[20] Yao Q.Z., Zhou Y.M., Sun Y.Q., Ye X.Y., J. Inorg. Organomet. P., 4 (2008), 477.

[21] Deng C., Yao N., Lu X., Qu S.X., Feng B., J. Mater. Sci., 16 (2009), 4394.

[22] Fu T., Zhao J.L., Wei J.H., Han Y., Xu K.W., J. Mater. Sci., 4 (2004), 1411.

[23] Annunziata M., Oliva A., Basile M.A., Giodano M., Mazzola N., J. Dent., 39 (2011), 720.

[24] Jackson A.W., Shebanova O., Hector A.L., Mcmillan P.F., J. Solid. State. Chem., 179 (2006), 1383.

[25] Zhou X.S., Peng F., Wang H.J., Yu H., Wang J., Mater. Res. Bull., 46 (2011), 840.

[26] Chen D., Li J.H., J. Phys. Chem. C, 114 (2010), 10478.

[27] SUn J.F., WANG M.Z., ZhaO Y.C., LI X.P., LIANG B. Y., J. Alloy. Compd., 482 (2009), 29.

[28] Galvanetto E., Galliano F.P., Borgioli F., BARdi U., LAVACCHI A., Thin Solid Films, 384 (2001), 223.

[29] Ismail I.M., Abdallah B., Abou-Kharroub M., Mrad O., Nucl. Instrum. Meth. B, 271 (2012), 102.

[30] Zhao J., Garza E.G., Lam K., Jones C.M., Appl. Surf. Sci., 158 (2000), 246.

[31] QIU Y.C., Yan K.Y., Yang S.H., Jin L.M., DENG H., LI W.S., ACS Nano, 4 (2010), 6515.

[32] Mazur M., KaczMareK D., DomaradzKi J., WojCIESZAK D., Mater. Sci.-Poland, 31 (2013), 71.

Received 2013-04-12 Accepted 2014-02-26 